\title{
The Banach fixed point theorem application to Hopf bifurcation of a generalized Boussinesq system
}

\author{
Junling Sun and Xuefeng Han*
}

\section{"Correspondence:}

hanxuefeng811015@163.com School of Mathematics and Information Science, Henan Polytechnic University, Jiaozuo, 454150, P.R. China

\begin{abstract}
This paper applies the Banach fixed point theorem to the study of the dynamical behavior of a three dimensional Boussinesq system with the temperature-dependent viscosity and thermal diffusivity under smooth external forces. We show that this system possesses time-periodic solutions bifurcating from a steady solution.
\end{abstract}

Keywords: Hopf bifurcation; periodic solution; Boussinesq systems

\section{Introduction}

The Banach fixed-point theorem is one of the most celebrated results in fixed-point theory and it states that a contraction on a complete metric space has a unique fixed point; its proof hinges on 'Picard iterations'. This method is applicable to a variety of subjects such as partial differential equations and engineering of image processing. In this paper, we apply the Banach fixed-point theorem to the study of the Hopf bifurcation of the Boussinesq system with the temperature-dependent viscosity and thermal diffusivity under external time-independent forces,

$$
\begin{aligned}
& U_{t}+(U \cdot \nabla) U-\nabla \cdot(v(T) \nabla U)+\nabla P=T e_{3}+f_{\alpha}, \\
& T_{t}+(U \cdot \nabla) T-\nabla \cdot(\kappa(T) \nabla T)=h_{\alpha}, \\
& \nabla \cdot U=0
\end{aligned}
$$

where $(t, x) \in \mathbf{R}^{+} \times \mathbf{R}^{3}, U$ is the velocity field, $T$ is the temperature function, the viscosity $v$ and the thermal diffusivity $\kappa$ depend on the temperature, $P$ denotes the pressure, the vector $e_{3}=(0,0,1), f_{\alpha}$, and $h_{\alpha}$ are external time-independent forces, which depend smoothly on some parameter $\alpha$. One may refer to the literature [1] for the derivation of this model and the related parameters.

The Boussinesq system is a very important model in fluid mechanics. It exhibits extremely rich phenomena. For example, Rayleigh-Bénard convection, geophysical fluid dynamics (see [2-6]). One of the key problems in the study of the dynamic behavior for the Boussinesq system is how to understand the bifurcation of solutions. To our knowledge, there are no results concerning the dynamical behavior of a Boussinesq system with $v$ and $\kappa$ depending on the temperature.

\section{Springer}

(c) 2015 Sun and Han; licensee Springer. This is an Open Access article distributed under the terms of the Creative Commons Attribution License (http://creativecommons.org/licenses/by/4.0), which permits unrestricted use, distribution, and reproduction in any medium, provided the original work is properly credited. 
We assume that $v(T)$ and $\kappa(T)$ satisfy

$$
\begin{aligned}
& v^{(k)}(\cdot), \kappa^{(k)}(\cdot)<0 \quad \text { for } k=0,1, \\
& v^{(k)}(\cdot)=\kappa^{(k)}(\cdot)=0 \quad \text { for } k=2,3, \ldots,
\end{aligned}
$$

where $\nu(\cdot)$ and $\kappa(\cdot)$ are smooth functions and are analytic at 0 .

Due to the technical restriction, two cases are still open. One is the case of $v^{(k)}(\cdot), \kappa^{(k)}(\cdot) \neq$ 0 for $k=2,3, \ldots$; the other case is that $v(T)$ and $\kappa(T)$ are not smooth functions. We also assume that external forces $f_{\alpha}$ and $h_{\alpha}$ can be chosen suitably such that $\left(U_{\alpha}(x)+U_{c_{1}}, T_{\alpha}(x)+\right.$ $\left.T_{c_{1}}, P_{\alpha}(x)\right)$ are a smooth solution of the steady Boussinesq system

$$
\begin{aligned}
& -\nabla \cdot(v(T) \nabla U)+(U \cdot \nabla) U+\nabla P=T e_{3}+f_{\alpha}, \\
& -\nabla \cdot(\kappa(T) \nabla T)+(U \cdot \nabla) T=h_{\alpha}, \\
& \nabla \cdot U=0,
\end{aligned}
$$

where $U_{c_{1}}=\left(c_{1}, 0,0\right)^{T}, T_{c_{1}}=\left(c_{1}, 0,0\right)^{T}$, and

$$
\lim _{|x| \rightarrow \infty} U_{\alpha}(x)=\mathbf{0}, \quad \lim _{|x| \rightarrow \infty} T_{\alpha}(x)=\mathbf{0} .
$$

Linearizing system (1.1)-(1.2) around the steady state $\left(U_{\alpha}(x)+U_{c_{1}}, T_{\alpha}(x)+T_{c_{1}}, P_{\alpha}(x)\right)$, we obtain

$$
\begin{aligned}
& u_{t}+c_{1} \partial_{x_{1}} u+u_{\alpha} \cdot \nabla u+u \cdot \nabla u_{\alpha}+u \cdot \nabla u-\nabla \cdot\left(v\left(v+T_{\alpha}+T_{c_{1}}\right) \nabla u\right)-\nabla p \\
& \quad-\nabla \cdot\left(\left(v\left(v+T_{\alpha}+T_{c_{1}}\right)-v\left(T_{\alpha}+T_{c_{1}}\right)\right) \nabla u_{\alpha}\right)-v e_{3}=0, \\
& v_{t}+c_{1} \partial_{x_{1}} v+T_{\alpha} \cdot \nabla v+u \cdot \nabla T_{\alpha}+u \cdot \nabla v-\nabla \cdot\left(\kappa\left(v+T_{\alpha}+T_{c_{1}}\right) \nabla v\right) \\
& \quad-\nabla \cdot\left(\left(\kappa\left(v+T_{\alpha}+T_{c_{1}}\right)-\kappa\left(T_{\alpha}+T_{c_{1}}\right)\right) \nabla T_{\alpha}\right)=0,
\end{aligned}
$$

with the incompressibility condition $\nabla \cdot u=0$. This condition leads to $\nabla \cdot\left(u u^{T}\right)=u \cdot \nabla u+$ $u \nabla \cdot u=u \cdot \nabla u$.

Thus it follows from (1.4)-(1.6) that

$$
\begin{aligned}
& u_{t}+c_{1} \partial_{x_{1}} u+v_{0} \Delta u+v_{0}^{\prime} c_{1} \partial_{x_{1} x_{1}} u+\nabla \cdot\left(u_{\alpha} u^{T}\right)+\nabla \cdot\left(u u_{\alpha}^{T}\right)+\nabla \cdot\left(u v^{T}\right)-\nabla p \\
& \quad-v_{0}^{\prime} \nabla \cdot\left(\left(v+T_{\alpha}\right) \nabla u\right)-v_{0}^{\prime} \nabla \cdot\left(v \nabla u_{\alpha}\right)-v e_{3}=0, \\
& v_{t}+c_{1} \partial_{x_{1}} v+\kappa_{0} \Delta v+\kappa_{0}^{\prime} c_{1} \partial_{x_{1} x_{1}} v+T_{\alpha} \cdot \nabla v+u \cdot \nabla T_{\alpha}+u \cdot \nabla v \\
& \quad+\kappa_{0}^{\prime} \nabla \cdot\left(\left(v+T_{\alpha}\right) \nabla v\right)-\kappa_{0}^{\prime} \nabla \cdot\left(v \nabla T_{\alpha}\right)=0 .
\end{aligned}
$$

The vorticity associated with the velocity field $u$ of the fluid is defined by $\omega=\nabla \times u$. Note that $\nabla \times \nabla \cdot\left(u u^{T}\right)=\nabla \cdot\left(\omega u^{T}-u \omega^{T}\right)$. We can obtain the vorticity equation from (1.7) as

$$
\begin{aligned}
\omega_{t} & +v_{0} \Delta \omega+c_{1} \partial_{x_{1}} \omega+v_{0}^{\prime} c_{1} \partial_{x_{1} x_{1}} \omega+\nabla \cdot\left(\omega_{\alpha} u^{T}-u_{\alpha} \omega^{T}\right)+\nabla \cdot\left(\omega u_{\alpha}^{T}-u \omega_{\alpha}^{T}\right) \\
& +\nabla \cdot\left(\omega u^{T}-u \omega^{T}\right)-v_{0}^{\prime} \nabla \cdot\left(\nabla \times\left(v+T_{\alpha}\right) \nabla u^{T}+\left(v+T_{\alpha}\right) \nabla \omega^{T}\right) \\
& -v_{0}^{\prime} \nabla \cdot\left((\nabla \times v) \nabla u_{\alpha}^{T}+v \nabla \omega_{\alpha}^{T}\right)-\partial_{x_{3}} v=0 .
\end{aligned}
$$


Note that the space of divergence free vector fields is invariant under the evolution (1.9). We can assume that $\nabla \cdot \omega=0$. Moreover, we can reconstruct the velocity $u$ from the vorticity $\omega$ by solving the equation

$$
\nabla \times u=\omega, \quad \nabla \cdot \omega=0 .
$$

On the other hand, the Biot-Savart law shows that the velocity field $u$ is defined in terms of the vorticity

$$
u(x)=-\frac{1}{4 \pi} \int_{\mathbf{R}^{3}} \frac{(x-y)^{\perp} \times \omega(y)}{|x-y|^{3}} d y, \quad x \in \mathbf{R}^{3} .
$$

Let $\varphi=(\omega, v)^{T}$. Then we can rewrite system (1.8)-(1.9) as the evolution equation

$$
\frac{d \varphi}{d t}+\mathcal{N} \varphi+G(\varphi)=F(\varphi)
$$

where

$$
\mathcal{N}=\left(\begin{array}{cc}
v_{0} \Delta+c_{1} \partial_{x_{1}}+v_{0}^{\prime} c_{1} \partial_{x_{1} x_{1}} & -\partial_{x_{3}} \\
0 & \kappa_{0} \Delta+c_{1} \partial_{x_{1}}+\kappa_{0}^{\prime} c_{1} \partial_{x_{1} x_{1}}
\end{array}\right)
$$

and

$$
G(\varphi)=\left(\begin{array}{l}
g_{1} \\
g_{2}
\end{array}\right), \quad F(\varphi)=\left(\begin{array}{l}
g_{3} \\
g_{4}
\end{array}\right)
$$

with

$$
\begin{aligned}
g_{1}= & \nabla \cdot\left(\omega_{\alpha} u^{T}-u_{\alpha} \omega^{T}-\omega u_{\alpha}^{T}+u \omega_{\alpha}^{T}-v_{0}^{\prime}\left(\nabla \times T_{\alpha}\right) \nabla u^{T}+v_{0}^{\prime} T_{\alpha} \nabla \omega^{T}\right. \\
& \left.-v_{0}^{\prime}(\nabla \times v) \nabla u_{\alpha}^{T}+v_{0}^{\prime} \nu \nabla \omega_{\alpha}^{T}\right), \\
g_{2}= & T_{\alpha} \cdot \nabla v+u \cdot \nabla T_{\alpha}-\kappa_{0}^{\prime} \nabla \cdot\left(v \nabla T_{\alpha}-T_{\alpha} \nabla v\right), \\
g_{3}= & -\nabla \cdot\left(\omega u^{T}-u \omega^{T}-v_{0}^{\prime}(\nabla \times v) \nabla u^{T}+v \nabla \omega^{T}\right), \\
g_{4}= & -u \cdot \nabla v-\kappa_{0}^{\prime} \nabla \cdot(v \nabla v) .
\end{aligned}
$$

To reach the essential spectrum of the operator $-(\widehat{\mathcal{N}}+\widehat{G})$ up to the imaginary axis, we need to make a similar assumption to [7]:

(H1) For any $\alpha \in\left[\alpha_{c}-\alpha_{0}, \alpha_{c}+\alpha_{0}\right], 0$ is not an eigenvalue of $\widehat{\mathcal{N}}+\widehat{G}$.

(H2) For $\alpha=\alpha_{c}$, the operator $-(\widehat{\mathcal{N}}+\widehat{G})$ has two pairs of eigenvalues $\left(\lambda_{0}^{+}, \mu_{0}^{+}\right)$and $\left(\lambda_{0}^{-}, \mu_{0}^{-}\right)$satisfying

$$
\begin{aligned}
& \lambda_{0}^{ \pm}\left(\alpha_{c}\right)=\mu_{0}^{ \pm}\left(\alpha_{c}\right)= \pm i \xi_{0} \neq 0, \quad \text { for } \xi_{0}>0, \\
& \left.\frac{d}{d \alpha} \operatorname{Re}\left(\lambda_{0}^{ \pm}(\alpha)\right)\right|_{\alpha=\alpha_{c}},\left.\quad \frac{d}{d \alpha} \operatorname{Re}\left(\mu_{0}^{ \pm}(\alpha)\right)\right|_{\alpha=\alpha_{c}}>0 .
\end{aligned}
$$

(H3) The remaining eigenvalue of $-(\widehat{\mathcal{N}}+\widehat{G})$ is strictly bounded away from the imaginary axis in the left half plane for all $\alpha \in\left[\alpha_{c}-\alpha_{0}, \alpha_{c}+\alpha_{0}\right]$. 
Under the generic assumption that the cubic coefficient terms $a_{1}, a_{2} \neq 0$ in (2.40)-(2.41), our main result is stated thus.

Theorem 1.1 Assume that (H1)-(H3) hold. Then system (1.1)-(1.3) has a one-dimensional family of small time-periodic solutions, i.e.

$$
U(x, t)=U\left(x, t+2 \pi / \xi_{1}\right), \quad T(x, t)=T\left(x, t+2 \pi / \xi_{2}\right)
$$

with $\alpha=\alpha_{c}+\epsilon, \epsilon \in\left(0, \alpha_{0}\right)$. Moreover, $\xi_{1}=\xi_{0}+\mathcal{O}(\epsilon), \xi_{2}=\xi_{0}+\mathcal{O}(\epsilon)$ and

$$
\|U(x, t)\|_{\mathbf{C}_{b}^{0}\left(\mathbf{R}^{3} \times\left[0,2 \pi / \xi_{1}\right]\right)}=\mathcal{O}(\epsilon), \quad\|T(x, t)\|_{\mathbf{C}_{b}^{0}\left(\mathbf{R}^{3} \times\left[0,2 \pi / \xi_{2}\right]\right)}=\mathcal{O}(\epsilon) .
$$

\section{Proof of Theorem 1.1}

We consider the following standard Sobolev space and spatially weighted Lebesgue space: $\mathbf{W}_{p}^{q}:=\left\{u:\|u\|_{p}^{q}:=\sum_{|\beta| \leq p}\left\|D^{\beta} u\right\|_{\mathbf{L}^{q}}^{q}<\infty\right\}, \mathbf{L}_{m}^{n}:=\left\{u:\|u\|_{m}^{n}:=\int_{\mathbf{R}^{3}} \rho^{m}(x) u^{n}(x) d x<\infty\right\}$ with the weighted function $\rho(x)=\sqrt{1+|x|^{2}}$. We denote $\mathbf{W}_{p}^{2}$ by $\mathbf{H}^{p}$ with the norm $\|u\|_{\mathbf{H}^{p}}=$ $\sum_{|\beta| \leq p}\left\|D^{\beta} u\right\|_{\mathbf{L}^{2}}^{2}$. When $q=2$, the Fourier transform is an isomorphism between $\mathbf{H}^{p}$ and $\mathbf{L}_{p}^{2}$ with $\|u\|_{\mathbf{L}_{p}^{2}}=\left\|\rho^{p} u\right\|_{\mathbf{L}^{2}}$. We also introduce the space $\mathbf{X}:=\left\{u=\left(u_{n}\right)_{n \in \mathbf{Z}}:\|u\|_{\mathbf{X}}<\infty\right\}$ and the weighted spaces $\mathcal{L}_{s}^{p}=\mathbf{L}_{s}^{p} \times \mathbf{L}_{s}^{p}, \mathcal{H}^{m}=\mathbf{H}^{m} \times \mathbf{H}^{m}, \mathcal{X}=\mathbf{X} \times \mathbf{X}$, which are equipped with the norms $\|u\|_{\mathbf{X}}=\sum_{n \in \mathbf{Z}}\left\|u_{n}\right\|_{\mathbf{H}^{m}},\|\varphi\|_{\mathcal{X}}:=\|u\|_{\mathbf{X}}+\|v\|_{\mathbf{X}},\|\varphi\|_{\mathcal{L}_{m}^{p}}:=\|u\|_{\mathbf{L}_{m}^{p}}+\|v\|_{\mathbf{L}_{m}^{p}}$, $\|\varphi\|_{\mathcal{H}^{m}}:=\|u\|_{\mathbf{H}^{m}}+\|v\|_{\mathbf{H}^{m}}$ for $\forall \varphi=(u, v)^{T} \in \mathcal{L}_{s}^{p}$ or $\mathcal{X}$.

Next, we look for $2 \pi$ time-periodic solutions of

$$
\Xi \frac{d \varphi}{d t}+\mathcal{N} \varphi+G(\varphi)=F(\varphi)
$$

where

$$
\Xi=\left(\begin{array}{cc}
\xi_{1} & 0 \\
0 & \xi_{2}
\end{array}\right), \quad \mathcal{N}=\left(\begin{array}{cc}
v_{0} \Delta+c_{1} \partial_{x_{1}}+v_{0}^{\prime} c_{1} \partial_{x_{1} x_{1}} & -\partial_{x_{3}} \\
0 & \kappa_{0} \Delta+c_{1} \partial_{x_{1}}+\kappa_{0}^{\prime} c_{1} \partial_{x_{1} x_{1}}
\end{array}\right)
$$

and

$$
G(\varphi)=\left(\begin{array}{l}
g^{1} \\
g^{2}
\end{array}\right), \quad F(\varphi)=\left(\begin{array}{l}
g^{3} \\
g^{4}
\end{array}\right)
$$

with

$$
\begin{aligned}
g^{1}= & \nabla \cdot\left(\omega_{\alpha} u^{T}-u_{\alpha} \omega^{T}-\omega u_{\alpha}^{T}+u \omega_{\alpha}^{T}-v_{0}^{\prime}\left(\nabla \times T_{\alpha}\right) \nabla u^{T}+v_{0}^{\prime} T_{\alpha} \nabla \omega^{T}\right. \\
& \left.-v_{0}^{\prime}(\nabla \times v) \nabla u_{\alpha}^{T}+v_{0}^{\prime} \nu \nabla \omega_{\alpha}^{T}\right), \\
g^{2}= & T_{\alpha} \cdot \nabla v+u \cdot \nabla T_{\alpha}-\kappa_{0}^{\prime} \nabla \cdot\left(\nu \nabla T_{\alpha}-T_{\alpha} \nabla v\right), \\
g^{3}= & -\nabla \cdot\left(\omega u^{T}-u \omega^{T}-v_{0}^{\prime}(\nabla \times v) \nabla u^{T}+v \nabla \omega^{T}\right), \\
g^{4}= & -u \cdot \nabla v-\kappa_{0}^{\prime} \nabla \cdot(v \nabla v) .
\end{aligned}
$$


According to the classical result in [8], we know that the essential spectrum of the operator $\mathcal{N}+G$ is a relatively compact perturbation of $\mathcal{N}$. It has the essential spectrum

$$
\begin{aligned}
& \operatorname{essspec}(\widehat{\mathcal{N}}) \\
& \quad=\left\{\lambda \in \mathcal{C}^{2}: \lambda=\left(-v_{0}|y|^{2}-v_{0}^{\prime} c_{1}\left|y_{1}\right|^{2}+i c y_{1},-\kappa_{0}|y|^{2}-\kappa_{0}^{\prime} c_{1}\left|y_{1}\right|^{2}+i c y_{1}\right), y \in \mathbf{R}^{3}\right\}
\end{aligned}
$$

Moreover, the spectra of $\mathcal{N}+G$ and $\mathcal{N}$ only differ by isolated eigenvalues of finite multiplicity. The above spectrum properties are critical to prove our main result.

For convenience, we rewrite (2.1) as

$$
\begin{aligned}
& \xi_{1} \omega_{t}=M_{1} \omega+g^{3}(\omega, u, v), \\
& \xi_{2} v_{t}=M_{2} v+g^{4}(\omega, u, v),
\end{aligned}
$$

where $g_{3}$ and $g_{4}$ defined in (2.2)-(2.3),

$$
\begin{aligned}
& M_{1} \omega=\overline{M_{1}} \omega+g^{1}=v_{0} \Delta \omega+v_{0}^{\prime} c_{1} \partial_{x_{1} x_{1}} \omega+c_{1} \partial_{x_{1}} \omega-\partial_{x_{3}} v+g^{1}, \\
& M_{2} v=\overline{M_{2}} v+g^{2}=\kappa_{0} \Delta v+\kappa_{0}^{\prime} c_{1} \partial_{x_{1} x_{1}} v+c_{1} \partial_{x_{1}} v+g^{2} .
\end{aligned}
$$

We make the ansatz $\omega(x, t)=\sum_{n \in \mathbf{Z}} \omega_{n}(x) e^{i n t}$ and $v(x, t)=\sum_{n \in \mathbf{Z}} v_{n}(x) e^{i n t}$ to (2.4)-(2.5). Then we obtain

$$
\begin{aligned}
& \left(i n \xi_{1}-M_{1}\right) \omega_{n}=g_{n}^{3}(\omega, u, v), \\
& \left(i n \xi_{2}-M_{2}\right) v_{n}=g_{n}^{4}(\omega, u, v),
\end{aligned}
$$

where $g^{3}(\omega, u, v)(x, t)=\sum_{n \in \mathbf{Z}} g_{n}^{3}(\omega, u, v) e^{i n t}, g^{4}(\omega, u, v)(x, t)=\sum_{n \in \mathbf{Z}} g_{n}^{4}(\omega, u, v) e^{i n t}$.

Note that we are interested in a real valued solution only. So we will always suppose that $\left(\omega_{n}, v_{n}\right)=\left(\omega_{-n}, v_{-n}\right)$ for $n \in \mathbf{Z}$. These series are uniformly convergent on $\mathbf{R}^{3} \times[0,2 \pi]$ in the spaces which we have chosen. More precisely, we have the following three results, which are taken from $[7,9]$.

Lemma 2.1 A linear operator $\mathbf{J}: \mathcal{X} \longrightarrow \mathbf{C}_{b}^{0}\left(\mathbf{R}^{3} \times[0, \pi], \mathbf{C}^{2}\right)$ is defined by

$$
(\mathbf{J} u)(x, t)=\tilde{u}(x, t):=\sum_{n \in \mathbf{Z}} u_{n}(x) e^{i n t}, \quad u=\left(u_{n}\right)_{n \in \mathbf{Z}} \in \mathcal{X} .
$$

Then $\mathbf{J}$ is bounded. Here $\mathbf{C}^{2}$ denotes the twice continuous differentiable function space.

Lemma 2.2 For $u=\left(u_{n}\right)_{n \in \mathbf{Z}}, v=\left(v_{n}\right)_{n \in \mathbf{Z}} \in \mathbf{X}$, the convolution $u * v \in \mathbf{X}$ is defined by

$$
(u * v)_{n}=\sum_{k \in \mathbf{Z}} u_{n-k} v_{k}, \quad n \in \mathbf{Z} .
$$

Then there exists $C>0$ such that $\|u * v\|_{\mathcal{X}} \leq C\|u\|_{\mathcal{X}}\|v\|_{\mathcal{X}}$.

Lemma 2.3 Let a linear operator $M: \mathbf{X} \longrightarrow \mathbf{X}$ be defined component-wise as $(M u)_{n}=$ $M_{n} u_{n}$ for $u=\left(u_{n}\right)_{n \in \mathbf{Z}}$. Then $\|M u\|_{\mathbf{X}}=\left(\left\|M_{0}\right\|_{\mathbf{H}^{m} \rightarrow \mathbf{H}^{m}}+\sup _{n \in \mathbf{Z} \backslash\{0\}}\|M\|_{\mathbf{H}^{m} \rightarrow \mathbf{H}^{m}}\right)\|u\|_{\mathbf{X}}$. 
By (H2) and (H3), we know that the operators $M_{1}$ and $M_{2}$ have two eigenvalues $\lambda_{0}^{ \pm}(\alpha)$ and $\mu_{0}^{ \pm}(\alpha)$, respectively, and all other eigenvalues of $M_{1}$ and $M_{2}$ are strictly bounded away from the imaginary axis in the left half plane. Thus we construct the $M_{j}$-invariant $(j=1,2)$ projections $\mathbf{P}_{ \pm 1, c}$ by

$$
\begin{aligned}
& P_{1, c} \omega=\left(\psi^{+, *}, \omega\right)_{\mathbf{L}^{2}} \psi^{+}, \quad P_{-1, c} \omega=\left(\psi^{-, *}, \omega\right)_{\mathbf{L}^{2}} \psi^{-}, \\
& P_{1, c} v=\left(\psi^{+, *}, v\right)_{\mathbf{L}^{2}} \psi^{+}, \quad P_{-1, c} v=\left(\psi^{-, *}, v\right)_{\mathbf{L}^{2}} \psi^{-},
\end{aligned}
$$

where $\psi^{ \pm}$denotes the associated normalized eigenfunctions, $\psi^{ \pm 1, *}$ denotes the associated normalized eigenfunctions of the adjoint operator $M_{j}^{*}$. The bounded 'stable' part of the projection is $\mathbf{P}_{ \pm 1, s}=I-\mathbf{P}_{ \pm 1, c}$, and we also know that $\mathbf{P}_{ \pm, c} M_{j}=M_{j} \mathbf{P}_{ \pm, c}$ and $\mathbf{P}_{ \pm, s} M_{j}=M_{j} \mathbf{P}_{ \pm, s}$. Thus we can split $\omega_{ \pm 1}$ and $v_{ \pm 1}$ as $\omega_{1}=\omega_{1, c}+\omega_{1, s}, \omega_{-1}=\omega_{-1, c}+\omega_{-1, s}, v_{1}=v_{1, c}+v_{1, s}$, and $v_{-1}=v_{-1, c}+v_{-1, s}$, where $\omega_{ \pm 1, c}=\mathbf{P}_{ \pm 1, c} \omega_{1}, \omega_{ \pm 1, s}=\mathbf{P}_{ \pm 1, s} \omega_{1}, v_{ \pm 1, c}=\mathbf{P}_{ \pm 1, c} v_{1}$, and $v_{ \pm 1, s}=\mathbf{P}_{ \pm 1, s} v_{1}$. Applying the above decompositions to (2.8)-(2.9), we have

$$
\begin{aligned}
& \left(i n \xi_{1}-M_{1}\right) \omega_{n}=g_{n}^{3}(\omega, u, v), \quad n= \pm 2, \pm 3, \ldots \\
& \left(i n \xi_{2}-M_{2}\right) v_{n}=g_{n}^{4}(\omega, u, v), \quad n= \pm 2, \pm 3, \ldots \\
& M_{1} \omega_{0}=g_{0}^{3}(\omega, u, v), \quad n=0 \\
& M_{2} v_{0}=g_{0}^{4}(\omega, u, v), \quad n=0, \\
& \left( \pm i \xi_{1}-M_{1}\right) \omega_{ \pm 1, s}=\mathbf{P}_{ \pm 1, s} g_{ \pm 1}^{3}(\omega, u, v) \\
& \left( \pm i \xi_{2}-M_{2}\right) v_{ \pm 1, s}=\mathbf{P}_{ \pm 1, s} g_{ \pm 1}^{4}(\omega, u, v) \\
& \left( \pm i \xi_{1}-M_{1}\right) \omega_{ \pm 1, c}=\mathbf{P}_{ \pm 1, c} g_{ \pm 1}^{3}(\omega, u, v) \\
& \left( \pm i \xi_{2}-M_{2}\right) v_{ \pm 1, c}=\mathbf{P}_{ \pm 1, c} g_{ \pm 1}^{4}(\omega, u, v)
\end{aligned}
$$

The organization of the proof of Theorem 1.1 is as follows: we first solve (2.14)-(2.15), then we use the fixed point theorem to solve (2.12)-(2.13) and (2.16)-(2.17). This is a nontrivial process due to the nonlinear terms $g_{n}^{3}(\omega, u, v)$ and $g_{n}^{4}(\omega, u, v)$. Finally, we employ the implicit function theorem to solve (2.18)-(2.19). The process of solving (2.18)-(2.19) is inspired by the classical Hopf-Bifurcation result [10].

Equations (2.12)-(2.19) can be rewritten as

$$
\begin{aligned}
& (i n \Xi+\mathcal{N}+G) \varphi_{n}=F_{n}(\varphi, u), \quad n= \pm 2, \pm 3, \ldots \\
& (\mathcal{N}+G) \varphi_{0}=F_{0}(\varphi, u), \quad n=0 \\
& ( \pm i \Xi+\mathcal{N}+G) \varphi_{ \pm 1, s}=\mathbf{P}_{ \pm 1, s} F_{ \pm 1}(\varphi, u) \\
& ( \pm i \Xi+\mathcal{N}+G) \varphi_{ \pm 1, c}=\mathbf{P}_{ \pm 1, c} F_{ \pm 1}(\varphi, u)
\end{aligned}
$$

Next we solve (2.21). The linear operator $\mathcal{N}$ has an essential spectrum up to the imaginary axis, and it is an invertible operator in the following sense. One may refer to [9] for the details of the proof.

Lemma 2.4 For $j=1,2$ and $f=\left(f^{1}, f^{2}\right)^{T} \in \mathcal{H}^{m-1} \cap \mathcal{L}^{1}$, the equation $\mathcal{N} \varphi=\partial_{j} f$ has a unique solution $\varphi=\mathcal{N}^{-1} \partial_{j} f \in \mathcal{H}^{m}$. Moreover, $\|\varphi\|_{\mathcal{H}^{m}} \leq C\|f\|_{\mathcal{H}^{m-1} \cap \mathcal{L}^{1}}$. 
This lemma tells us that $\widehat{\mathcal{N}}\left(i y_{i}, i y_{i}\right)^{T}$ is a bounded compact operator from $\mathcal{L}_{m}^{2}$ to itself. Furthermore, the spectra of $\widehat{\mathcal{N}}+\widehat{G}$ and $\widehat{\mathcal{N}}$ only differ by isolated eigenvalues of finite multiplicity (see the book of Henry [8, p.136]). The following three lemmas give the solvability of (2.21); they are similar to Lemma 6-8 in [9]. Here we omit their proofs.

Lemma 2.5 Assume that $(\mathrm{H} 1)-(\mathrm{H} 3)$ hold. Then (2.21) has a unique solution $\varphi_{0}=(\mathcal{N}+$ $G)^{-1} F_{0}(\varphi, u)$. Moreover, $\left\|\varphi_{0}\right\|_{\mathcal{H}^{m}} \leq C\left\|y_{j}^{-1} I_{2 \times 2} \widehat{F_{0}}(\hat{\varphi}, \hat{u})\right\|_{\mathcal{L}_{m}^{2}}$, where $I_{2 \times 2}$ and $\widehat{F_{0}}$ denote the $2 \times$ 2 unit matrix and the application of Fourier transform to $F_{0}$, respectively.

Lemma 2.6 There exists a constant $C>0$ such that

$$
\|u\|_{\mathbf{H}^{m}} \leq C\|\omega\|_{\mathbf{H}^{m}}, \quad\left\|\partial_{x_{i}} u\right\|_{\mathbf{H}^{m}} \leq C\|\omega\|_{\mathbf{H}^{m}} .
$$

Lemma 2.7 For $m>\frac{3}{2}$, there exists a positive constant $C$ such that

$$
\|\hat{\omega} * \hat{u}\|_{\mathbf{L}_{m}^{2}} \leq C\|\hat{\omega}\|_{\mathbf{L}_{m}^{2}}\|\hat{u}\|_{\mathbf{L}_{m}^{2}}
$$

Applying the Fourier transform to $g^{3}$ and $g^{4}$ in (2.2)-(2.3), we get

$$
\begin{aligned}
& \widehat{g^{3}}=-i y\left(\hat{\omega} * \hat{u}^{T}-\hat{u} * \hat{\omega}^{T}+v_{0}^{\prime}|y|^{2} \hat{v} * \hat{u}^{T}+i y \hat{v} * \hat{\omega}\right), \\
& \widehat{g^{4}}=-i y \hat{u} * \hat{v}-2 \kappa_{0}^{\prime}|y|^{2} \hat{v} * \hat{v} .
\end{aligned}
$$

From the form of the nonlinear terms $g^{3}$ and $g^{4}$, it is critical to estimate the term as $u v$ and $u^{2}$. For convenience, we derive some estimates as regards the nonlinear terms $N^{1}(\varphi)=\varphi^{2}$ and $N^{2}(\varphi, \psi)=\varphi \psi$. Since the proof of the next lemma is similar to Lemma 4 in [7], we omit it.

Lemma 2.8 Define $N^{1}: \mathcal{X} \longrightarrow \mathcal{X}$ by $N^{1}(\varphi)_{n}=N_{n}^{1}(\mathrm{~J} \varphi)$ and $N^{2}: \mathcal{X} \times \mathcal{X} \longrightarrow \mathcal{X}$ by $N^{2}(\varphi)_{n}=$ $N_{n}^{2}(\mathbf{J} \varphi, \mathbf{J} \psi)$ for $\varphi, \psi \in \mathcal{X}$. Then we have

$$
\left\|N^{1}(\varphi)\right\|_{\mathcal{X}} \leq C\|\varphi\|_{\mathcal{X}}^{2}, \quad\left\|N^{2}(\varphi, \psi)\right\|_{\mathcal{X}} \leq C\|\psi\|_{\mathcal{X}}\|\varphi\|_{\mathcal{X}}
$$

for $\varphi, \psi \in \mathcal{X}$ with $\|\varphi\|_{\mathcal{X}} \leq 1$ and $\|\psi\|_{\mathcal{X}} \leq 1$. Moreover, we have

$$
\begin{aligned}
&\left\|N^{1}\left(\varphi^{1}\right)-N^{1}\left(\varphi^{2}\right)\right\|_{\mathcal{X}} \leq C\left(\left\|\varphi^{1}\right\|_{\mathcal{X}}+\left\|\varphi^{2}\right\|_{\mathcal{X}}\right)\left\|\varphi^{1}-\varphi^{2}\right\|_{\mathcal{X}} \\
&\left\|N^{2}\left(\varphi^{1}, \psi^{1}\right)-N^{2}\left(\varphi^{2}, \psi^{2}\right)\right\|_{\mathcal{X}} \leq C\left(\left\|\varphi^{1}\right\|_{\mathcal{X}}+\left\|\varphi^{2}\right\|_{\mathcal{X}}+\left\|\psi^{1}\right\|_{\mathcal{X}}+\left\|\psi^{2}\right\|_{\mathcal{X}}\right) \\
& \times\left(\left\|\varphi^{1}-\varphi^{2}\right\|_{\mathcal{X}}+\left\|\psi^{1}-\psi^{2}\right\|_{\mathcal{X}}\right),
\end{aligned}
$$

for $\varphi^{1}, \varphi^{2}, \psi^{1}, \psi^{2} \in \mathcal{X}$ with $\left\|\varphi^{1}\right\|_{\mathcal{X}},\left\|\varphi^{2}\right\|_{\mathcal{X}},\left\|\psi^{1}\right\|_{\mathcal{X}},\left\|\psi^{2}\right\|_{\mathcal{X}} \leq 1$

By a small modification of the proof of Lemma 7 in [7], we have the following result.

Lemma 2.9 Assume that $\xi$ is close enough to $\xi_{0}$. Then there exists a constant $C>0$ such that

$$
\left\|\left(i n \xi_{i}-\overline{M_{i}}\right)^{-1}\right\|_{\mathbf{H}^{m} \rightarrow \mathbf{H}^{m}} \leq C, \quad\left\|\left(i n \xi_{i}-\overline{M_{i}}\right)^{-1} \nabla^{j} \cdot\right\|_{\mathbf{H}^{m} \rightarrow \mathbf{H}^{m}} \leq C,
$$




$$
\begin{aligned}
& \left\|\left(i n \xi_{i}-M_{i}\right)^{-1}\right\|_{\mathbf{H}^{m} \rightarrow \mathbf{H}^{m}} \leq C, \quad\left\|\left(i n \xi_{i}-M_{i}\right)^{-1} \nabla^{j} \cdot\right\|_{\mathbf{H}^{m} \rightarrow \mathbf{H}^{m}} \leq C, \\
& \left\|\left(i n \xi_{i}-M_{i}\right)^{-1} \mathbf{P}_{ \pm 1, s}\right\|_{\mathbf{H}^{m} \rightarrow \mathbf{H}^{m}} \leq C, \quad\left\|\left(i n \xi_{i}-M_{i}\right)^{-1} \nabla^{j} \cdot \mathbf{P}_{ \pm 1, s}\right\|_{\mathbf{H}^{m} \rightarrow \mathbf{H}^{m}} \leq C,
\end{aligned}
$$

for $n \neq 0$ and $j=1,2$. Here $\overline{M_{i}}$ and $M_{i}$ are defined in (2.6)-(2.7), respectively.

Thus by Lemma 2.9, we can rewrite (2.20) and (2.22) as

$$
\begin{aligned}
& \varphi_{n}=(i n \Xi+\mathcal{N}+G)^{-1} F_{n}(\varphi, u), \quad n= \pm 2, \pm 3, \ldots \\
& \varphi_{ \pm 1, s}=( \pm i \Xi+\mathcal{N}+G)^{-1} \mathbf{P}_{ \pm 1, s} F_{ \pm 1}(\varphi, u)
\end{aligned}
$$

i.e.

$$
\begin{aligned}
& \omega_{n}=\left(i n \xi_{1}-M_{1}\right)^{-1} g_{n}^{3}(\omega, u, v), \quad n= \pm 2, \pm 3, \ldots \\
& v_{n}=\left(i n \xi_{2}-M_{2}\right)^{-1} g_{n}^{4}(\omega, u, v), \quad n= \pm 2, \pm 3, \ldots \\
& \omega_{ \pm 1, s}=\left( \pm i \xi_{1}-M_{1}\right)^{-1} \mathbf{P}_{ \pm 1, s} g_{ \pm 1}^{3}(\omega, u, v) \\
& v_{ \pm 1, s}=\left( \pm i \xi_{2}-M_{2}\right)^{-1} \mathbf{P}_{ \pm 1, s} g_{ \pm 1}^{4}(\omega, u, v)
\end{aligned}
$$

Using Lemmas 2.8 and 2.9, we obtain the solvability of (2.26)-(2.29). Since the proof is similar to [9], we omit it.

Lemma 2.10 Assume that there exist $\sigma_{1}, \sigma_{2}>0$ such that for all $\xi_{1}, \xi_{2}>0$ with $\mid \xi_{1}-$ $\xi_{0}|,| \xi_{2}-\xi_{0} \mid \leq \sigma_{1}$ and all $\omega_{ \pm 1, c}, v_{ \pm 1, c} \in \mathbf{H}^{m}$ with $\left\|\omega_{ \pm 1, c}\right\|_{\mathbf{H}^{m}},\left\|\nu_{ \pm 1, c}\right\|_{\mathbf{H}^{m}} \leq \sigma_{2}$. Then (2.26)(2.29) has a unique solution $(\tilde{\omega}, \tilde{v})=\Phi\left(\omega_{c}, v_{c}\right) \in \mathcal{X}$, where $\omega_{c}=\left(\omega_{-1, c}, \omega_{1, c}\right), v_{c}=\left(v_{-1, c}, v_{1, c}\right)$, $\tilde{\omega}=\left(\ldots, \omega_{-2}, \omega_{-1, c}+\omega_{-1, s}, \omega_{0}, \omega_{1, c}+\omega_{1, s}, \omega_{2}, \ldots\right), \tilde{v}=\left(\ldots, v_{-2}, v_{-1, c}+v_{-1, s}, v_{0}, v_{1, c}+v_{1, s}, v_{2}, \ldots\right)$. Moreover, there exists a positive $C$ such that

$$
\begin{aligned}
& \Phi(0,0)=(0,0), \quad\left\|\tilde{\omega}-\omega_{c}\right\|_{\mathbf{X}} \leq C\left(\left\|\omega_{-1, c}\right\|_{\mathbf{H}^{m}}^{2}+\left\|\omega_{1, c}\right\|_{\mathbf{H}^{m}}^{2}\right), \\
& \left\|\tilde{v}-v_{c}\right\|_{\mathbf{X}} \leq C\left(\left\|v_{-1, c}\right\|_{\mathbf{H}^{m}}^{2}+\left\|v_{1, c}\right\|_{\mathbf{H}^{m}}^{2}\right),
\end{aligned}
$$

with $\tilde{\omega}-\omega_{c}:=\left(\ldots, 0, \omega_{-1, c}, 0, \omega_{1, c}, 0, \ldots\right)$ and $\tilde{v}-v_{c}:=\left(\ldots, 0, v_{-1, c}, 0, v_{1, c}, 0, \ldots\right)$.

Proof For fixed $\xi_{1}, \xi_{2}>0$ so close to $\xi_{0}$ and given $\omega_{ \pm 1, c}, v_{ \pm 1, c} \in \mathbf{H}^{m}$ with $\left\|\omega_{ \pm 1, c}\right\|_{\mathbf{H}^{m}}$, $\left\|v_{ \pm 1, c}\right\|_{\mathbf{H}^{m}} \leq \sigma_{2}$. Define the operator

$$
\begin{aligned}
\Gamma:\left(\tilde{\omega}^{*}, \tilde{v}^{*}\right) & \longmapsto(\tilde{\omega}, \tilde{v}) \\
& =\left(\tilde{\omega}^{*}+\left(\ldots, 0, \omega_{-1, c}, 0, \omega_{1, c}, 0, \ldots\right), \tilde{v}^{*}+\left(\ldots, 0, v_{-1, c}, 0, v_{1, c}, 0, \ldots\right)\right) \\
& \longmapsto(\omega, v) \longmapsto\left(\tilde{\omega}^{* *}, \tilde{v}^{* *}\right)=\text { the right hand side of }(2.26)-(2.29),
\end{aligned}
$$

where $(\omega, v)=(\mathbf{J} \tilde{\omega}, \mathbf{J} \tilde{v})$ are defined in Lemma 2.1 and

$$
\begin{aligned}
\left(\tilde{\omega}^{*}, \tilde{v}^{*}\right) & =\left(\left(\ldots, \omega_{-2}, \omega_{-1, s}, \omega_{0}, \omega_{1, s}, \omega_{2}, \ldots\right),\left(\ldots, v_{-2}, v_{-1, s}, v_{0}, v_{1, s}, v_{2}, \ldots\right)\right), \\
(\tilde{\omega}, \tilde{v})= & \left(\tilde{\omega}^{*}+\omega_{c}, \tilde{v}^{*}+v_{c}\right) \\
= & \left(\tilde{\omega}^{*}+\left(\ldots, 0, \omega_{-1, c}, 0, \omega_{1, c}, 0, \ldots\right), \tilde{v}^{*}+\left(\ldots, 0, v_{-1, c}, 0, v_{1, c}, 0, \ldots\right)\right) .
\end{aligned}
$$


Now we prove the operator $\Gamma$ is a self-map of a sufficiently small ball in $\mathcal{X}$. By Lemmas 2.62.9 and the form of the nonlinear terms $\widehat{g^{3}}, \widehat{g^{4}}$ in (2.24)-(2.25), we derive

$$
\begin{aligned}
\left\|\tilde{\omega}^{* *}\right\|_{\mathbf{X}} \leq & C \sup \left\{\left\|\left(i n \xi_{1}-M_{1}\right)^{-1}\right\|_{\mathbf{H}^{m} \rightarrow \mathbf{H}^{m}},\left\|\left( \pm i \xi_{1}-M_{1}\right)^{-1} \mathbf{P}_{ \pm 1, s}\right\|_{\mathbf{H}^{m} \rightarrow \mathbf{H}^{m}}\right. \\
& \left\|\left(i n \xi_{1}-M_{1}\right)^{-1} \nabla^{j}\right\|_{\mathbf{H}^{m} \rightarrow \mathbf{H}^{m}},\left\|\left( \pm i \xi_{1}-M_{1}\right)^{-1} \nabla^{j} \mathbf{P}_{ \pm 1, s}\right\|_{\mathbf{H}^{m} \rightarrow \mathbf{H}^{m}} \\
& : n \in \mathbf{Z} \backslash\{-1,1\}\} \times\left\|\left(\tilde{g}_{n}^{3}(\omega, u, v)\right)_{n \in \mathbf{Z}}\right\|_{\mathbf{X}} \\
\leq & C\left\|\tilde{g}^{3}(\tilde{\omega}, u, \tilde{v})\right\|_{\mathbf{X}} \\
\leq & C\left(\|\tilde{\omega}\|_{\mathbf{X}}\|u\|_{\mathbf{X}}+\|\tilde{\omega}\|_{\mathbf{X}}\|\tilde{v}\|_{\mathbf{X}}+\|u\|_{\mathbf{X}}\|\tilde{v}\|_{\mathbf{X}}\right) \\
\leq & C\left(\|\tilde{\omega}\|_{\mathbf{X}}^{2}+\|\tilde{\omega}\|_{\mathbf{X}}\|\tilde{v}\|_{\mathbf{X}}\right) \\
\leq & C\left(\left\|\tilde{\omega}^{*}\right\|_{\mathbf{X}}^{2}+\left\|\omega_{-1, c}\right\|_{\mathbf{H}^{m}}^{2}+\left\|\omega_{1, c}\right\|_{\mathbf{H}^{m}}^{2}+\left\|\tilde{v}^{*}\right\|_{\mathbf{X}}^{2}+\left\|v_{-1, c}\right\|_{\mathbf{H}^{m}}^{2}+\left\|v_{1, c}\right\|_{\mathbf{H}^{m}}^{2}\right) \\
\leq & C\left(\left\|\tilde{\omega}^{*}\right\|_{\mathbf{X}}^{2}+\left\|\tilde{v}^{*}\right\|_{\mathbf{X}}^{2}+\sigma_{2}^{2}\right), \\
\left\|\tilde{v}^{* *}\right\|_{\mathbf{X}} \leq & C \sup \left\{\left\|\left(i n \xi_{2}-M_{2}\right)^{-1}\right\|_{\mathbf{H}^{m} \rightarrow \mathbf{H}^{m}},\left\|\left( \pm i \xi_{2}-M_{2}\right)^{-1} \mathbf{P}_{ \pm 1, s}\right\|_{\mathbf{H}^{m} \rightarrow \mathbf{H}^{m}},\right. \\
& \left\|\left(i n \xi_{2}-M_{2}\right)^{-1} \nabla^{j}\right\|_{\mathbf{H}^{m} \rightarrow \mathbf{H}^{m}},\left\|\left( \pm i \xi_{2}-M_{2}\right)^{-1} \nabla^{j} \mathbf{P}_{ \pm 1, s}\right\|_{\mathbf{H}^{m} \rightarrow \mathbf{H}^{m}} \\
& : n \in \mathbf{Z} \backslash\{-1,1\}\} \times\left\|\left(\tilde{g}_{n}^{4}(\omega, u, v)\right)_{n \in \mathbf{Z}}\right\|_{\mathbf{X}} \\
\leq & C\left\|\tilde{g}^{4}(\tilde{\omega}, u, \tilde{v})\right\|_{\mathbf{X}} \\
\leq & C\left(\|\tilde{v}\|_{\mathbf{X}}^{2}+\|\tilde{\omega}\|_{\mathbf{X}}\|\tilde{v}\|_{\mathbf{X}}\right) \\
\leq & C\left(\left\|\tilde{\omega}^{*}\right\|_{\mathbf{X}}^{2}+\left\|\omega_{-1, c}\right\|_{\mathbf{H}^{m}}^{2}+\left\|\omega_{1, c}\right\|_{\mathbf{H}^{m}}^{2}+\left\|\tilde{v}^{*}\right\|_{\mathbf{X}}^{2}+\left\|v_{-1, c}\right\|_{\mathbf{H}^{m}}^{2}+\left\|v_{1, c}\right\|_{\mathbf{H}^{m}}^{2}\right) \\
\leq & C\left(\left\|\tilde{\omega}^{*}\right\|_{\mathbf{X}}^{2}+\left\|\tilde{v}^{*}\right\|_{\mathbf{X}}^{2}+\sigma_{2}^{2}\right),
\end{aligned}
$$

where

$$
\begin{aligned}
& \tilde{g}^{3}=2 \omega \cdot u^{T}+v_{0}^{\prime} \nu \cdot u^{T}+v \cdot \omega^{T}, \\
& \tilde{g}^{4}=u \cdot v^{T}+2 \kappa_{0}^{\prime} \nu \cdot v^{T} .
\end{aligned}
$$

Thus, for $\sigma_{2} \leq \frac{1}{\sqrt{2 C}}$ and $\left(\tilde{\omega}^{*}, \tilde{v}^{*}\right) \in \mathcal{X}$ with $\left\|\left(\tilde{\omega}^{*}, \tilde{v}^{*}\right)\right\|_{\mathcal{X}} \leq \frac{1}{\sqrt{2 C}}$, we have

$$
\begin{aligned}
\left\|\Gamma\left(\tilde{\omega}^{*}, \tilde{v}^{*}\right)\right\|_{\mathcal{X}} & =\left\|\tilde{\omega}^{* *}\right\|_{\mathcal{X}}+\left\|\tilde{v}^{* *}\right\|_{\mathbf{X}} \\
& \leq C\left(\left(\left\|\tilde{\omega}^{*}\right\|_{\mathbf{X}}+\left\|\tilde{v}^{*}\right\|_{\mathbf{X}}\right)^{2}+\sigma_{2}^{2}\right) \leq 1,
\end{aligned}
$$

which implies that for sufficient small $\sigma_{2}>0$. Hence, by the Banach fixed point theorem, we obtain a unique fixed point $\left(\tilde{\theta}^{*}, \tilde{v}^{*}\right) \in \mathcal{X}$ of $\Gamma$, which means that (2.26)-(2.29) have a solution of $(\tilde{\omega}, \tilde{v})=\left(\tilde{\omega}^{*}+\omega_{c}, \tilde{v}^{*}+v_{c}\right)$. Moreover, if $\left(\omega_{ \pm 1, c}, v_{ \pm 1, c}\right)=(0,0)$, then $\Phi(0,0)=(0,0)$. Next we prove the second inequality in $(2.30)$ and $(2.31)$. Note that $\left(\tilde{\omega}^{*}, \tilde{v}^{*}\right)=\Gamma\left(\tilde{\omega}^{*}, \tilde{v}^{*}\right)=$ $\left(\tilde{\theta}^{* *}, \tilde{v}^{* *}\right)$. This, combined with (2.32)-(2.33), allows us to derive

$$
\begin{aligned}
\left\|\tilde{\omega}-\omega_{c}\right\|_{\mathbf{X}} & =\left\|\tilde{\omega}^{*}\right\|_{\mathbf{X}}=\left\|\tilde{\omega}^{* *}\right\|_{\mathbf{X}} \\
& \leq C\left(\left\|\tilde{\omega}^{*}\right\|_{\mathbf{X}}^{2}+\left\|\omega_{-1, c}\right\|_{\mathcal{H}^{m}}^{2}+\left\|\omega_{1, c}\right\|_{\mathcal{H}^{m}}^{2}\right),
\end{aligned}
$$




$$
\begin{aligned}
\left\|\tilde{v}-v_{c}\right\|_{\mathbf{X}} & =\left\|\tilde{v}^{*}\right\|_{\mathbf{X}}=\left\|\tilde{v}^{* *}\right\|_{\mathbf{X}} \\
& \leq C\left(\left\|\tilde{v}^{*}\right\|_{\mathbf{X}}^{2}+\left\|v_{-1, c}\right\|_{\mathcal{H}^{m}}^{2}+\left\|v_{-1, c}\right\|_{\mathcal{H}^{m}}^{2}\right) .
\end{aligned}
$$

Thus we deduce that for a sufficient small ball $\mathbf{B}_{r}(0) \subset \mathbf{B}_{1}(0)$,

$$
\begin{aligned}
& \left\|\tilde{\omega}-\omega_{c}\right\|_{\mathbf{X}} \leq C\left(\left\|\omega_{-1, c}\right\|_{\mathbf{H}^{m}}^{2}+\left\|\omega_{1, c}\right\|_{\mathbf{H}^{m}}^{2}\right), \\
& \left\|\tilde{v}-v_{c}\right\|_{\mathbf{X}} \leq C\left(\left\|v_{-1, c}\right\|_{\mathbf{H}^{m}}^{2}+\left\|v_{1, c}\right\|_{\mathbf{H}^{m}}^{2}\right),
\end{aligned}
$$

where

$$
\begin{aligned}
& \tilde{\omega}-\omega_{c}:=\left(\ldots, 0, \omega_{-1, c}, 0, \omega_{1, c}, 0, \ldots\right), \\
& \tilde{v}-v_{c}:=\left(\ldots, 0, v_{-1, c}, 0, v_{1, c}, 0, \ldots\right) .
\end{aligned}
$$

This completes the proof.

It remains to analyze (2.18)-(2.19). We restate the equations:

$$
\begin{aligned}
& \left( \pm i \xi_{1}-M_{1}\right) \omega_{ \pm 1, c}=\mathbf{P}_{ \pm 1, c} g_{ \pm 1}^{3}(\omega, u, v), \\
& \left( \pm i \xi_{2}-M_{2}\right) v_{ \pm 1, c}=\mathbf{P}_{ \pm 1, c} g_{ \pm 1}^{4}(\omega, u, v) .
\end{aligned}
$$

It follows from $\left(\omega_{-1}, v_{-1}\right)=\left(\overline{\omega_{1}}, \overline{v_{1}}\right)$ and $\left(g_{-1}^{3}, g_{-1}^{4}\right)=\left(\overline{g_{1}^{3}}, \overline{g_{1}^{4}}\right)$ that the '-' equation is the complex conjugate of the ' + ' equation. By Lemma 2.1, we can denote $(\omega, v)=(\mathbf{J} \tilde{\omega}, \mathbf{J} \tilde{v})$ by means of $(\tilde{\omega}, \tilde{v})=\Phi\left(\omega_{c}, v_{c}\right)=\Phi\left(\left(\overline{\omega_{1, c}}, \omega_{1, c}\right),\left(\overline{v_{1, c}}, v_{1, c}\right)\right)$. Our target is to find $\left(\xi_{1}, \alpha\right)$ and $\left(\xi_{2}, \alpha\right)$ close to $\left(\xi_{0}, \alpha_{c}\right)$ and a nontrivial solution $\left(\omega_{1, c}, v_{1, c}\right)=\left(\omega_{1, c}, v_{1, c}\right)(x)$ of

$$
\begin{aligned}
& -i \xi_{1} \omega_{1, c}+M_{1} \omega_{1, c}+\mathbf{P}_{1, c} g_{1}^{3}\left(\mathbf{J} \Phi\left(\overline{\omega_{1, c}}, \omega_{1, c}, \overline{\nu_{1, c}}, v_{1, c}\right)\right)=0, \\
& -i \xi_{2} v_{1, c}+M_{2} v_{1, c}+\mathbf{P}_{1, c} g_{1}^{4}\left(\mathbf{J} \Phi\left(\overline{\omega_{1, c}}, \omega_{1, c}, \overline{v_{1, c}}, v_{1, c}\right)\right)=0 .
\end{aligned}
$$

Since $\omega_{1, c}, v_{1, c} \in \mathrm{C} \psi^{+}$and $\left(M_{1} \psi^{+}, M_{2} \psi^{+}\right)=\left(\lambda_{0}^{+}(\alpha) \psi^{+}, \mu_{0}^{+}(\alpha) \psi^{+}\right)$, we can write $\omega_{1, c}=\eta \psi^{+}$ and $v_{1, c}=\delta \psi^{+}$. Then by (2.34)-(2.35), for some $\eta, \delta \in \mathrm{C} \backslash\{0\}$, we obtain

$$
\begin{aligned}
& -i \xi_{1} \eta \psi^{+}+\lambda_{0}^{+}(\alpha) \eta \psi^{+}+\mathbf{P}_{1, c} g_{1}^{3}\left(\mathbf{J} \Phi\left(\overline{\eta \psi^{+}}, \eta \psi^{+}, \overline{\delta \psi^{+}}, \delta \psi^{+}\right)\right)=0, \\
& -i \xi_{2} \delta \psi^{+}+\mu_{0}^{+}(\alpha) \delta \psi^{+}+\mathbf{P}_{1, c} g_{1}^{4}\left(\mathbf{J} \Phi\left(\overline{\eta \psi^{+}}, \eta \psi^{+}, \overline{\delta \psi^{+}}, \delta \psi^{+}\right)\right)=0 .
\end{aligned}
$$

Now we introduce $\left(p_{1, c}, \theta_{1, c}\right)$ by $\left(\mathbf{P}_{1, c} \omega, \mathbf{P}_{1, c} v\right)=\left(p_{1, c}(\omega) \psi^{+}, \theta_{1, c}(v) \psi^{+}\right)$. Then (2.36)-(2.37) can be simplified to

$$
\begin{aligned}
& -i \xi_{1} \eta+\lambda_{0}^{+}(\alpha) \eta+g^{3}(\alpha, \eta, \delta)=0, \quad \text { for some } \eta \in \mathrm{C}, \\
& -i \xi_{2} \delta+\mu_{0}^{+}(\alpha) \delta+g^{4}(\alpha, \eta, \delta)=0, \quad \text { for some } \delta \in \mathrm{C},
\end{aligned}
$$

where the cubic coefficient $a_{1} \neq 0$ and $a_{2} \neq 0$ in

$$
g^{3}(\alpha, \eta, \delta):=p_{1, c}\left(g_{1}^{3}\left(\mathbf{J} \Phi\left(\overline{\eta \psi^{+}}, \eta \psi^{+}, \overline{\delta \psi^{+}}, \delta \psi^{+}\right)\right)\right)
$$




$$
g^{4}(\alpha, \eta, \delta):=\theta_{1, c}\left(g_{1}^{4}\left(\mathbf{J} \Phi\left(\overline{\eta \psi^{+}}, \eta \psi^{+}, \overline{\delta \psi^{+}}, \delta \psi^{+}\right)\right)\right)
$$

Note that $\left|p_{1, c}(\omega)\right| \leq C\left\|\mathbf{P}_{1, c} \omega\right\|_{\mathbf{H}^{m}} \leq C\|\omega\|_{\mathbf{H}^{m}}$ and $\left|\theta_{1, c}(v)\right| \leq C\left\|\mathbf{P}_{1, c} v\right\|_{\mathbf{H}^{m}} \leq C\|v\|_{\mathbf{H}^{m}}$. Thus by (2.30)-(2.31) and (2.40)-(2.41), we derive

$$
\begin{aligned}
& \left|p_{1, c}\left(g_{1}^{3}\left(\mathbf{J} \Phi\left(\overline{\eta \psi^{+}}, \eta \psi^{+}, \overline{\delta \psi^{+}}, \delta \psi^{+}\right)\right)\right)\right| \leq C\left(|\eta|^{2}+|\delta|^{2}\right), \\
& \left|\theta_{1, c}\left(g_{1}^{4}\left(\mathbf{J} \Phi\left(\overline{\eta \psi^{+}}, \eta \psi^{+}, \overline{\delta \psi^{+}}, \delta \psi^{+}\right)\right)\right)\right| \leq C\left(|\eta|^{2}+|\delta|^{2}\right),
\end{aligned}
$$

where we use the following relation: $(\tilde{\omega}, \tilde{v})=\Phi\left(\omega_{c}, v_{c}\right)=\Phi\left(\overline{\eta \psi^{+}}, \eta \psi^{+}, \overline{\delta \psi^{+}}, \delta \psi^{+}\right)$.

Analogously to the case of the classical Hopf-bifurcation result [10], the Banach fixed point theorem can be applied to (2.38)-(2.39), as soon as the zero solution is divided out. It is sufficient to find the real value solutions (i.e. $\left.\left(\gamma_{1}, \gamma_{2}\right)=(\eta, \delta) \in \mathbf{R}^{2}\right)$ of (2.38)-(2.39). Hence we define the complex-valued smooth function

$$
\begin{aligned}
& \Upsilon^{1}\left(\gamma_{1}, \gamma_{2} ; \varrho, \epsilon\right):= \begin{cases}-i\left(\xi_{0}+\varrho\right)+\lambda_{0}^{+}\left(\alpha_{c}+\epsilon\right)+\gamma_{1}^{-1} g^{3}\left(\alpha_{c}+\epsilon, \gamma_{1}, \gamma_{2}\right), & \gamma_{1} \neq 0, \\
-i\left(\xi_{0}+\varrho\right)+\lambda_{0}^{+}\left(\alpha_{c}+\epsilon\right), & \gamma_{1}=0,\end{cases} \\
& \Upsilon^{2}\left(\gamma_{1}, \gamma_{2} ; \varrho, \epsilon\right):= \begin{cases}-i\left(\xi_{0}+\varrho\right)+\mu_{0}^{+}\left(\alpha_{c}+\epsilon\right)+\gamma_{2}^{-1} g^{4}\left(\alpha_{c}+\epsilon, \gamma_{1}, \gamma_{2}\right), & \gamma_{2} \neq 0, \\
-i\left(\xi_{0}+\varrho\right)+\mu_{0}^{+}\left(\alpha_{c}+\epsilon\right), & \gamma_{2}=0 .\end{cases}
\end{aligned}
$$

It follows from $\left(\lambda_{0}^{+}\left(\alpha_{c}\right), \mu_{0}^{+}\left(\alpha_{c}\right)\right)=\left(i \xi_{0}, i \xi_{0}\right)$ that $\left(\Upsilon^{1}(0,0,0,0), \Upsilon^{2}(0,0,0,0)\right)=(0,0)$. Moreover, by assumption (H2) for the Jacobi matrix

$$
\begin{aligned}
& \left.\mathbf{D}_{\rho, \epsilon} \Upsilon^{1}\left(\gamma_{1}, \gamma_{2} ; \varrho, \epsilon\right)\right|_{\gamma_{1}=\gamma_{2}=\varrho=\epsilon=0}=\left(\begin{array}{cc}
0 & \left.\frac{d}{d \beta} \operatorname{Re} \lambda_{0}^{+}(\alpha)\right|_{\alpha=\alpha_{c}} \\
-1 & \left.\frac{d}{d \beta} \operatorname{Im} \lambda_{0}^{+}(\alpha)\right|_{\alpha=\alpha_{c}}
\end{array}\right), \\
& \left.\mathbf{D}_{\rho, \epsilon} \Upsilon^{2}\left(\gamma_{1}, \gamma_{2} ; \varrho, \epsilon\right)\right|_{\gamma_{1}=\gamma_{2}=\varrho=\epsilon=0}=\left(\begin{array}{cc}
0 & \left.\frac{d}{d \beta} \operatorname{Re} \mu_{0}^{+}(\alpha)\right|_{\alpha=\alpha_{c}} \\
-1 & \left.\frac{d}{d \beta} \operatorname{Im} \mu_{0}^{+}(\alpha)\right|_{\alpha=\alpha_{c}}
\end{array}\right)
\end{aligned}
$$

with respect to $\rho, \epsilon$ one has $\left.\operatorname{det} \mathbf{D}_{\rho, \epsilon} \Upsilon^{1}\left(\gamma_{1}, \gamma_{2} ; \varrho, \epsilon\right)\right|_{\gamma_{1}=\gamma_{2}=\varrho=\epsilon=0}=\left.\frac{d}{d \beta} \operatorname{Re} \lambda_{0}^{+}(\alpha)\right|_{\alpha=\alpha_{c}}>0$ and $\left.\operatorname{det} \mathbf{D}_{\rho, \epsilon} \Upsilon^{2}\left(\gamma, \gamma_{2} ; \varrho, \epsilon\right)\right|_{\gamma_{1}=\gamma_{2}=\varrho=\epsilon=0}=\left.\frac{d}{d \beta} \operatorname{Re} \mu_{0}^{+}(\beta)\right|_{\alpha=\alpha_{c}}>0$. Thus for sufficient small $\gamma_{1}, \gamma_{2}>0$, we can find a function $\gamma_{1} \mapsto\left(\varrho\left(\gamma_{1}\right), \epsilon\left(\gamma_{1}\right)\right)$ and $\gamma_{2} \mapsto\left(\varrho\left(\gamma_{2}\right), \epsilon\left(\gamma_{2}\right)\right)$ with $\varrho(0)=\epsilon(0)=0$ such that

$$
\begin{aligned}
& -i\left(\xi_{0}+\varrho\left(\gamma_{1}\right)\right)+\lambda_{0}^{+}\left(\alpha_{c}+\epsilon\left(\gamma_{1}\right)\right)-\gamma_{1}^{-1} g^{3}\left(\alpha_{c}+\epsilon\left(\gamma_{1}\right), \gamma_{1}, \alpha_{c}+\epsilon\left(\gamma_{2}\right), \gamma_{2}\right)=0, \\
& -i\left(\xi_{0}+\varrho\left(\gamma_{2}\right)\right)+\mu_{0}^{+}\left(\alpha_{c}+\epsilon\left(\gamma_{2}\right)\right)-\gamma_{2}^{-1} g^{4}\left(\alpha_{c}+\epsilon\left(\gamma_{1}\right), \gamma_{1}, \alpha_{c}+\epsilon\left(\gamma_{2}\right), \gamma_{2}\right)=0 .
\end{aligned}
$$

Note the degree of nonlinearity. Then it follows from differentiating this equation that $\epsilon^{(i)} \neq 0$ for some first $i$. Hence the function $\gamma_{1} \mapsto \epsilon\left(\gamma_{1}\right)$ and $\gamma_{1} \mapsto \epsilon\left(\gamma_{2}\right)$ can locally be inverted to yield a function $\epsilon \mapsto \gamma_{1}(\epsilon)$ and $\epsilon \mapsto \gamma_{2}(\epsilon)$. This implies that the following equation holds:

$$
\begin{aligned}
& -i\left(\xi_{0}+\varrho\left(\gamma_{1}(\epsilon)\right)\right) \gamma_{1}(\epsilon)+\lambda_{0}^{+}\left(\alpha_{c}+\epsilon\right) \gamma_{1}(\epsilon)-g^{3}\left(\alpha_{c}+\epsilon, \gamma_{1}(\epsilon), \gamma_{2}(\epsilon)\right)=0, \\
& -i\left(\xi_{0}+\varrho\left(\gamma_{2}(\epsilon)\right)\right) \gamma_{2}(\epsilon)+\mu_{0}^{+}\left(\alpha_{c}+\epsilon\right) \gamma_{2}(\epsilon)-g^{4}\left(\alpha_{c}+\epsilon, \gamma_{1}(\epsilon), \gamma_{2}(\epsilon)\right)=0,
\end{aligned}
$$

for sufficient small $\epsilon>0$. 
Therefore we obtain the desired solutions of (2.34)-(2.35) by setting $\left(\xi_{1}, \xi_{2}\right)=\left(\xi_{0}+\right.$ $\left.\varrho\left(\gamma_{1}(\epsilon)\right), \xi_{0}+\varrho\left(\gamma_{2}(\epsilon)\right)\right), \alpha=\alpha_{c}+\epsilon$, and $\left(\omega_{1, c}, v_{1, c}\right)=\left(\gamma_{1}(\epsilon) \psi_{\alpha_{c}+\epsilon}^{+}, \gamma_{2}(\epsilon) \psi_{\alpha_{c}+\epsilon}^{+}\right)(x)$. This result combined with Lemmas 3.2, 3.8, and (1.10) gives the proof of Theorem 1.1.

\section{Competing interests}

The authors declare that they have no competing interests.

\section{Authors' contributions}

All authors contributed equally to the writing of this paper. All authors read and approved the final manuscript.

\section{Acknowledgements}

The authors expresses his sincere thanks to the anonymous referees for a very careful reading and for providing many valuable comments and suggestions which led to an improvement of this paper.

Received: 20 September 2014 Accepted: 25 December 2014 Published online: 12 February 2015

\section{References}

1. Turcotte, DL, Schubert, G: Geodynamics: Applications of Continuum Physics to Geological Problems. Wiley, New York (1982)

2. Bodenschatz, E, Pesch, W, Ahlers, G: Recent developments in Rayleigh-Bénard convection. In: Annual Review of Fluid Mechanics. Annu. Rev. Fluid Mech., vol. 32, pp. 709-778. Annual Reviews, Palo Alto (2000)

3. Getling, AV: Rayleigh-Bénard Convection. Structures and Dynamics. Advanced Series in Nonlinear Dynamics, vol. 11. World Scientific, River Edge (1998)

4. Lions, JL, Temam, R, Wang, S: On the equations of large-scale ocean. Nonlinearity 5, 1007-1053 (1992)

5. Pedlosky, J: Geophysical Fluid Dynamics, 2nd edn. Springer, New York (1987)

6. Tritton, DJ: Physical Fluid Dynamics, 2nd edn. Oxford Science Publications. Clarendon, New York (1988)

7. Brand, T, Kunze, M, Schneider, G, Seelbach, T: Hopf bifurcation and exchange of stability in diffusive media. Arch. Ration. Mech. Anal. 171, 263-296 (2004)

8. Henry, D: Geometric Theory of Semilinear Parabolic Equations. Springer, New York (1981)

9. Yan, WP: Hopf-Bifurcation theorem and stability for the Magneto-Hydrodynamics equations. Topol. Methods Nonlinear Anal. (to appear)

10. Marsden, JE, Mccracken, M: The Hopf Bifurcation and Its Applications. Springer, Berlin (1976)

\section{Submit your manuscript to a SpringerOpen ${ }^{\circ}$ journal and benefit from:}

- Convenient online submission

Rigorous peer review

- Immediate publication on acceptance

- Open access: articles freely available online

- High visibility within the field

- Retaining the copyright to your article 